[Agr. Biol. Chem., Vol. 33, No. 9, p. 1295 1300, 1969]

\title{
Synthesis of Oligosaccharides by Growing Culture of Leuconostoc mesenteroides
}

\author{
Part IV. ${ }^{*}$ Oligosaccharide Formation in the Presence \\ of Various Types of Glucobioses as Acceptors
}

\author{
By Fumio Yamauchi and Yhuji OHwada** \\ Department of Agricultural Chemistry, Tohoku University, Sendai \\ Received March 3, 1969
}

\begin{abstract}
With all glucobioses (eleven types) as acceptors, Leuconostoc mesenteroides (NRRL B-5I2) was grown on a sucrose medium. The trisaccharides produced were analyzed for their yields, and the trisaccharide structures were determined after separation on a column.

The yield of the tri- and higher-saccharides indicated that isomaltose $(28 \%)$ was the most efficient acceptor and maltose $(24 \%)$ was next. With the other eight glucobioses, oligosaccharides were obtained in $3 \sim 15 \%$ yield. Among these sugars, $\alpha, \beta$-trehalose (15\%) and $\beta, \beta$-trehalose $(11 \%)$ were efficient acceptors next to maltose, but $\alpha, \alpha$-trehalose was inert.

In every case, except for cellobiose, $\alpha$-glucosyl transfer occurred to the position 6 of non-reducing moiety of glucobiose.

The sugars produced contained five new trisaccharides which were isolated as pure compounds.
\end{abstract}

Koepsell et al." showed that oligosaccharides were formed by the action of dextransucrase of Leuc. mesenteroides on sucrose and certain sugars, notably maltose and isomaltose. According to Baily et al. ${ }^{2}$ a branched trisaccharide, $2^{G}$ - $\alpha$-glucosyllactose was produced when the dextran-producing organism Betacoccus arabinosus was grown in a medium containing sucrose and lactose, and a similar trisaccharide, $2^{1}-\alpha$-glucosylcellobiose, was produced in a medium of sucrose and cellobiose. Neely ${ }^{31}$ reported that a tetrasaccharide, $2^{G}-\alpha-$ glucosylraffinose, was produced by dextran-

* Part III, K. Aso and F. Yamauchi, Agr. Biol. Chem., 25, 10 (1961).

** Present address; Nisshin Seito Company, Tokyo.

1) H. J. Koepsell, H. M. Tsuchiya, N. M. Hell-

man, A. Kazenko, C. A. Hoffman, E. S. Sharpe and

R. W. Jackson, J. Biol. Chem., 200, 793 (1952).

2) R. W. Baily, S. A. Barker, E. J. Bourne, P. M. Grant and M. Stacey, J. Chem. Sac., 1958, 1959.

3) W. B. Neely, Arch. Biochem. Biophys., 79, 154 (1959). sucrase in a mixture of sucrose and raffinose. Suzuki and Hehre ${ }^{4}$ found that Leuc. mesenteroides synthesized a non-reducing trisaccharide, lactulosucrose, by $\alpha$-glucosyl transfer from sucrose to lacturose.

The above studies are classified into two types of transglucosylation with glucosyl moiety as an acceptor. In one type, the transferred glucosyl group enters at position 6 of the non-reducing moiety of acceptors such as maltose and isomaltose. The other type is the transfer of glucosyl group to position 2 of the reducing moiety in cellobiose and lactose, and the oligosaccharide produced has a branched structure.

It is of interest to determine the relationship between the structure of the acceptor and that of the resulting oligosaccharide, together with the properties of the new oligo-

4) H. Suzuki and E.J. Hehre, Arch. Biochem. Biophys., 105, 339 (1964). 
saccharide produced other than those described above. Therefore, we used all of the possible glucobioses as acceptors, and determined the structure of the oligosaccharides isolated from the growing-culture medium of Leuc. mesenteroides $\mathrm{B}-512$.

\section{MATERIALS AND METHODS}

Glucobioses, $\quad \alpha, \alpha$-Trehalose $2 \mathrm{H}_{2} \mathrm{O}, \mathrm{mp} 98^{\circ} \mathrm{C}$ and cellobiose, $\mathrm{mp} 225^{\circ} \mathrm{C}$ were obtained from commercial sources. $\alpha, \beta$-Trehalose $\mathrm{H}_{2} \mathrm{O}, \mathrm{mp} 145^{\circ} \mathrm{C}$ (octaacetate, $\operatorname{mp} 178^{\circ} \mathrm{C}$ ) was synthesized chemically. ${ }^{5 /} \alpha$-Kojibiose $\mathrm{H}_{2} \mathrm{O}, \mathrm{mp} 187^{\circ} \mathrm{C}$ and nigerose, amorphous ( $\beta$-octaacetate, $m p 150^{\circ} \mathrm{C}$ ) were prepared by acetolysis of a dextran. ${ }^{6)} \alpha$-Sophorose $\mathrm{H}_{2} \mathrm{O}, \mathrm{mp} 195^{\circ} \mathrm{C}$ was prepared by chemical synthesis.7 $\beta$-Laminaribiose, mp $183^{\circ} \mathrm{C}$ was prepared by acetolysis of pachyman. ${ }^{81} \quad \beta$-Maltose $\mathrm{H}_{2} \mathrm{O}$ was purified from commercial preparation on a carbon column. Isomaltose, amorphus ( $\beta$-octaacetate, $\mathrm{mp} 144^{\circ} \mathrm{C}$ ) and $\beta$-gentiobiose, $\mathrm{mp} 185^{\circ} \mathrm{C}$ ( $\alpha$-octaacetate, $\mathrm{mp} 191^{\circ} \mathrm{C}$ ) were isolated from a hydrol's on a carbon column. The purity of each glucobiose was checked by means of paper chromatography and paper electrophoresis.

Paper chromatography and paper electrophoresis Paper chromatographic separations were made in pyridine: $n$-butanol:water (4:6:3, by vol.) (a), and isopropanol: $n$-butanol water (7:1:2, by vol.) (b). Rf values were shown with solvent (a) on a four multiple chromatogram by the ascending method.

Paper electrophoresis in borate buffer ( $\mathrm{pH} 10)^{10,11}$ was carried out with Toyo filter paper No. 51 ( $40 x$ $16 \mathrm{~cm}$ ) at $600 \mathrm{~V}$ for $3 \mathrm{hr}$. Paper electrophoresis in molybdate solution $(\mathrm{pH} 5.5)^{12}$ was made with Toyo filter paper No. $51(40 \times 11 \mathrm{~cm})$ at $100 \mathrm{~V}$ for $1 \mathrm{hr}$ in a water-cooled apparatus. $M_{G}$ values show the mo-

5) B. Helferich and $\mathrm{K}$. Weis, Ber., 89, 314 (1956). 6) K. Matsuda, H. Watanabe, K. Fujimoto and K. Aso. Nature, 191, 278 (1961).

7) B. Coxon and H. G. Fletcher, Jr., J. Org. Chem. 26, 2892 (1961).

8) K. Fujimoto, K. Matsuda and K. Aso, Nippon Nogeikagaku Kaishi, 36, 346 (1962).

9) K. Aso and K. Shibasaki, ibid., 29, 856 (1955).

10) A. B. Foster, J. Chem. Soc., 1953, 982.

11) K. Aso and S. Hamada, J. Fermentation Tech. Japan., 33, 45 (1955).

12) E.J. Bourne, D. H. Hutson and H. Weigel, J. Chem. Soc., 1960, 4252. bilites with respect to glucose in the borate buffer, and $M_{S}$ values are with respect to sorbitol in the molybdate solution.

The reagents used to detect the compounds were aniline hydrogen phthalate (1) for reducing sugars, silver nitrate-sodium hydroxide (2) for non-reducing and reducing sugars, and resorcinol-trichloroacetic acid (3) for ketoses.

Reduction of sugars with sodium borohydride. Reduced sugars were prepared by reduction of free sugars $(0.2 \%)$ with sodium borohydride $(0.2 \%)$ in an aqueous solution at room temperature overnight, followed by de-ionization with Amberlite IR-120 and repeated distillation with anhydrous methanol.

Quantitative determination of oligosaccharide. The zone of paper containing each oligosaccharide on a three multiple chromatogram with solvent (a) was cut off by use of the guide strips and the oligosaccharide extracted with water was analyzed by the anthrone method.13

Oligosaccharide synthesis in a glucobiose and sucrose medium. Sterile medium $(10 \mathrm{ml})$ contained yeast extract $(0.196)$, potassium monohydrogen phosphate $(0.5 \%)$, sodium chloride $(0.1 \%)$, ammonium sulfate $(0.06 \%)$, hydrated magnesium sulfate $(0.02 \%)$, sucrose $(2 . \%)$, and glucobiose $(1.0 \mathrm{~g}, 10 \%$ as anhydrosugar $)$. The medium was inoculated with Leuc. mesenteroides NRRL B-5l2 and incubated for 6 days during which the $\mathrm{pH}$ fell from 6.0 to $4.0 \sim 5.0$.

Separation of oligosaccharides on a carbon column. Oligosaccharides were fractionated on a column (2.5X $23 \mathrm{~cm}$ ) containing carbon $(15 \mathrm{~g})$ and celite $(15 \mathrm{~g})$ with occasional test by the Molisch reaction. When the three trehaloses and $\alpha$-linked sugars were used in the media, fractionation of mono-, di-, tri- and highersaccharides was performed with water $(300 \mathrm{ml}), 3 \sim$ $5 \%$ ethanol $(420 \mathrm{ml}), 9 \sim 10 \%$ ethanol $(360 \mathrm{ml})$ and $20 \%$ ethanol $(360 \mathrm{ml})$, respectively. In the case of the $\beta$-linked glucobiose-containing media, fractionation of mono-, di-, tri- and higher-saccharides was made with water $(300 \mathrm{ml}), 7 \sim 9 \%$ ethanol $(420 \mathrm{ml}), 13 \sim$ $17 \%$ ethanol $(360 \mathrm{ml})$ and $30 \%$ ethanol $(360 \mathrm{ml})$, re spectively.

13) R.J. Dimler, W. C. Schaefer, C.S. Wise, C. E. Rist, Anal. Chem., 24, 1411 (1952). 
Hydrolysis of trisaccharides with acid and emulsin. Complete hydrolysis of trisaccharides $(2 \mathrm{mg})$ was performed with $1.5 \mathrm{~N}$ hydrochloric acid at $100^{\circ} \mathrm{C}$ for $3 \mathrm{hr}$. Partial hydrolysis of trisaccharides $(3 \sim 5 \mathrm{mg})$ was carried out with $0.1 \mathrm{~N}$ hydrochloric acid at $100^{\circ} \mathrm{C}$ for 30 and $60 \mathrm{~min}$ (the trisaccharide from $\alpha, \beta$-trehalose) and for 60 and $120 \mathrm{~min}$ (other trisaccharides). Reduced trisaccharides ( $3 \mathrm{mg}$ ) were hydrolyzed partially with $0.1 \mathrm{~N}$ hydrochloric acid at $100^{\circ} \mathrm{C}$ for 60 and $120 \mathrm{~min}$. Sugars (1 mg) were hydrolyzed with an almond $\beta$-glucosidase, ${ }^{14}$ emulsin, in acetate buffer $(0.5 \mathrm{ml}, \mathrm{pH} 4.7)$ at $30^{\circ} \mathrm{C}$ for 2 days. Under these conditions the pure isomaltose was left intact, but gentiobiose was completely hydrolyzed to glucose.

\section{RESULTS}

Paper chromatography of each medium showed the formation of oligosaccharides

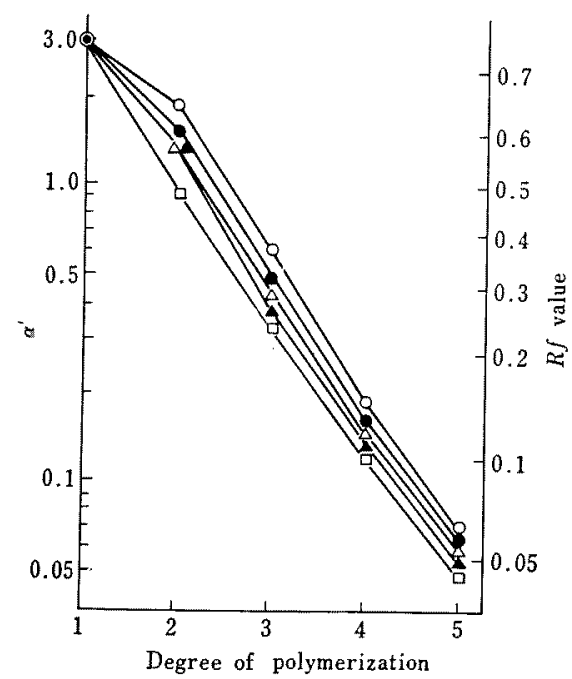

FIG. 1. Paper Chromatographic Mobility of Oligosaccharides Produced from $\alpha$-Linked Disaccharides as Acceptor.

$$
\begin{aligned}
& -\bigcirc-\text { Glucose } \bigcirc-01,3-\alpha \quad \square-1,4-\alpha \\
& \Delta-\Delta 1,2-\alpha \quad \Delta-1,1-\alpha, \beta \quad \square-\square 1,6-\alpha \\
& \alpha^{\prime}=R f /(1-R f) \text {; chromatographic solvent, pyri- } \\
& \text { dine: } n \text {-butanol: water (4:6:3 by vol.) with four } \\
& \text { multiple development. }
\end{aligned}
$$

14) H. Tauber, f. Biol. Chem., 99, 257 (1932). which moved slower than the acceptor disaccharides. Only from $\alpha, \alpha$-trehalose was detected no oligosaccharide. When the concentration of $\alpha, \alpha$-trehalose was increased to $20 \%$ in the medium, no oligosaccharide was observed either. The produced oligosaccharides could be detected almost to the extent of pentasaccharides on a paper chromatogram. $R f$ values of the produced oligosaccharides are shown in Figs. 1 and 2. The trisaccharides gave the largest spots among the produced oligosaccharides and the amount of other oligosaccharides decreased with the increasing degree of polymerization.

The oligosaccharides produced from $\alpha, \beta$ - and $\beta, \beta$-trehalose were detected with the reagent (2), while no reaction was shown with reagent (1) like the $\alpha, \beta$ - and $\beta, \beta$-trehalose. Therefore, these were non-reducing sugars. With reagent (1), the oligosaccharides produced from koji-

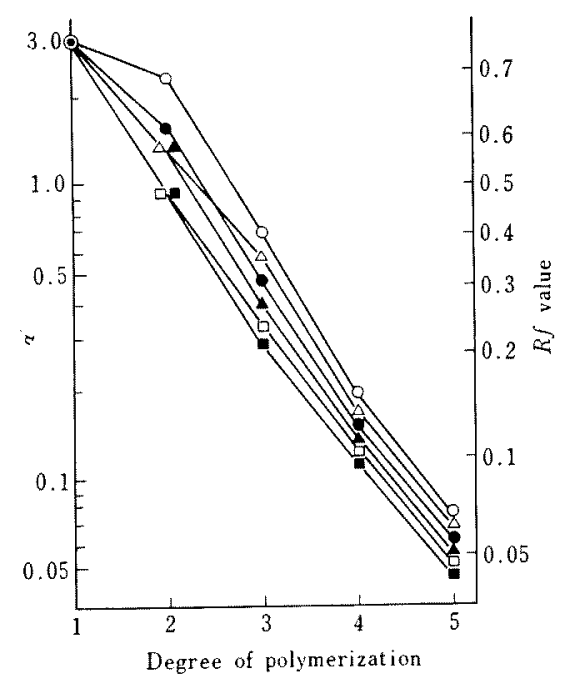

FIG. 2. Paper Chromatographic Mobility of Oligosaccharides Produced from $\beta$-Linked Disaccharides as Acceptor.

$$
\begin{array}{lll}
-\odot-\text { Glucose } & \bigcirc-\bigcirc 1,3-\beta & -\bullet 1,4-\beta \\
\Delta-\triangle 1,2-\beta & \mathbf{A}-1,1-\beta, \beta & (\square-\square 1,6-\alpha) \\
\square-\mathbf{B} 1,6-\beta & &
\end{array}
$$

Chromatographic methods are the same as those described in Fig. 1. 
Table I. Structure, Yield, and $[\alpha]_{D}$ of Produced Trisaccharide

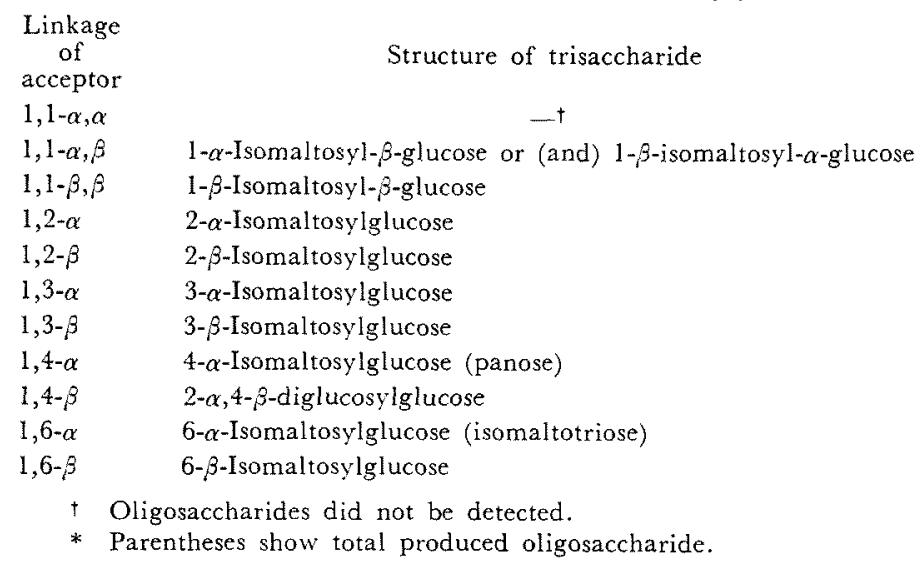

$\begin{array}{cc}\begin{array}{c}\text { Yield to } \\ \text { acceptor }\end{array} & {[\alpha]_{D}^{12} \text { in water }} \\ (\%) & - \\ -\dagger & -\dagger \\ 12.9(15.4) * & +133 \mathrm{C}, 1.4 \\ 9.5(10.9) & +42 \mathrm{C}, 1.5 \\ 4.5(8.0) & +148 \mathrm{C}, 1.8 \\ 4.8(6.2) & +81 \mathrm{C}, 0.4 \\ 3.1(4.6) & +155 \mathrm{C}, 0.7 \\ 2.5(3.0) & +88 \mathrm{C}, 0.7 \\ 16.5(24.0) & +150 \mathrm{C}, 2.0 \\ 3.0(4.3) & +98 \mathrm{C}, 0.9 \\ 23.4(28.0) & +144 \mathrm{C}, 2.0 \\ 9.7(10.5) & +65 \mathrm{C}, 1.8\end{array}$

biose gave a red color like kojibiose $e^{151}$ and the oligosaccharides produced from sophorose showed a reddish brown color like sophorose. ${ }^{151}$ When detected with reagent (3), the oligosaccharides produced in every medium gave no spots. It proved that a transfructosylation to the acceptor did not occur.

Quantitative yields of oligosaccharides to the various acceptors are shown in Table I. The yields of trisaccharide and higher oligosaccharides from $1.00 \mathrm{~g}$ of each acceptor after separation on a column were as follows: 86 and $31 \mathrm{mg}$ from $\alpha, \beta$-trehalose, 65 and $8 \mathrm{mg}$ from $\beta, \beta$-trehalose, 39 and $28 \mathrm{mg}$ from kojibiose, 32 and $13 \mathrm{mg}$ from sophorose, 28 and $15 \mathrm{mg}$ from nigerose, 19 and $12 \mathrm{mg}$ from laminaribiose, 115 and $78 \mathrm{mg}$ from maltose, 21 and $6 \mathrm{mg}$ from cellobiose, 158 and $48 \mathrm{mg}$ from isomaltose, and 80 and $12 \mathrm{mg}$ from gentiobiose. Each trisaccharide eluted from the column showed a single spot and the higher oligosaccharides to the extent of hexasaccharides could be detected more clearly by chromatography with solvent (a).

The chromatographic mobilities of the oligosaccharides in a homologous series showed

15) K. Aso, F. Yamauchi and K. Matsuda, $J$. Fermentation Tech. Japan, 117, (1955). some important relationships ${ }^{16,17)}$ with respect to their degree of polymerization. In Figs. I and $2, \log \alpha^{\prime}\left(\alpha^{\prime}=R f /(1-R f)\right)$ of the oligosaccharide produced are plotted against their expected degree of polymerization. Each $\alpha^{\prime}$ series from disaccharide to pentasaccharide nearly paralleled that of the isomaltoseries oligosaccharides, with the one exception of the cellobiose-containing trisaccharide. From these results, it appeared that a successive 1, 6- $\alpha$ glucosylation to the acceptor occurred.

With respect to the trisaccharides, the above suggestion was confirmed by partial acid hydrolysis. In a partial hydrolyzate of each trisaccharide, the presence of isomaltose together with acceptor (glucobiose) was shown by paper chromatography and paper electrophoresis, except only for the trisaccharides from cellobiose which showed kojibiose and cellobiose.

A linkage joined to reducing glucose was suggested by the paper electrophoresis in the borate buffer ${ }^{10,111}$ and the molybdate solution, ${ }^{121}$ because the mobility of an oligosaccharide was affected mainly on that linkage. All

16) D. French and G. M. Wild, J.Am. Chem. Soc., 75,2612 (1953).

17) K. Aso and F. Yamauchi, J. Fermentation Tech. Japan, 33, 194 (1955). 
Table II. Mobilities of Disaccharides and the Produced TRISACCHARIDES BY PAPER ELECTROPHORESIS

$\begin{array}{lcccc}\begin{array}{c}\text { Sugar } \\ \text { (acceptor) }\end{array} & \begin{array}{c}\text { Bisacch. } \\ \left(M_{G}\right)\end{array} & \begin{array}{c}\text { Trisacch. } \\ \left(M_{G}\right)\end{array} & \begin{array}{c}\text { Molybdate soln., pH 5.5 } \\ \text { Disacch. } \\ \left(M_{S}\right)\end{array} & \begin{array}{c}\text { Trisacch. } \\ \left(M_{S}\right)\end{array} \\ \alpha, \alpha \text {-Trehalose } & 0.10 & 0.1 & 0.0 & \text { * }^{*} \\ \alpha,-\beta \text {-Trehalose } & 0.10 & 0.1 & 0.0 & 0.0 \\ \beta, \beta \text {-Trehalose } & 0.10 & 0.2 & 0.0 & 0.0 \\ \text { Kojibiose } & 0.31 & 0.15 & 0.7 & 0.6 \\ \text { Sophorose } & 0.30 & 0.55 & 0.7 & 0.6 \\ \text { Nigerose } & 0.67 & 0.55 & 0.0 & 0.0 \\ \text { Laminaribiose } & 0.69 & 0.2 & 0.0 & 0.0 \\ \text { Maltose } & 0.33 & 0.15 & 0.5 & 0.4 \\ \text { Cellobiose } & 0.27 & 0.55 & 0.7 & 0.0 \\ \text { Isomaltose } & 0.67 & 0.55 & 0.7 & 0.6 \\ \text { Gentiobiose } & 0.71 & & & \end{array}$

types of glucobioses could be distinguished by use of these electrophoresis, although not distinguished in the anomeric configuration. The mobilities of disaccharides (acceptors) and the trisaccharides by paper electrophoresis were shown in Table II. Mobilities of glucobioses in borate buffer are divided into three groups; a lower mobility of 1,1-linkages, a middle mobility of 1,2- and 1,4-linkages, and a higher mobility of $1,3-$ and 1,6-linkages. On the other hand mobilities between 1,2and 1,4-linkages, and between 1,3- and 1,6linkages can be distinguished from each other with molybdate solution as shown in Table II. A comparison of the mobility of disaccharide with that of trisaccharide in borate buffer showed that the trisaccharide moved with a small decrease of about 0.1 in $M_{G}$ value depending on the molecular size, but essentially the same as the disaccharide. Paper electrophoresis of disaccharide and trisaccharides in molybdate solution also showed essentially the same mobility, with one exception of the trisaccharide containing cellobiose residue, negative movement of which indicated also an evidence for a branched structure. These results showed that the linkage at the reducing end in the trisaccharide structure was the same as that of the disac- charide used as an acceptor.

Confirmative evidence was obtained by partial hydrolysis of the reduced trisaccharides retained on partial acid hydrolysis of the reduced trisaccharides show a linkage at the non-reducing end in trisaccharide molecules. Isomaltose was found in every partial hydrolyzate, accordingly, the linkage at the reducing end was 1,6- $\alpha$ linkage, and the linkage provided from acceptor occurred at the reducing end. In the reduced trisaccharide from cellobiose no reducing disaccharide was detected. This was a further evidence for the branched structure.

The difference in two trisaccharides isolated from isomaltose and gentiobiose was shown by the action of emulsin. The one from isomaltose was inert with emulsin, but the other from gentiobiose was hydrolyzed to isomaltose and glucose; this was shown on a paper chromatogram.

Higher specific rotations of trisaccharides than that of each disaccharide in Table I also indicated that the transglucosylation in the form of the $\alpha$-configuration occurred.

The experiments described above lead to the conclusion on the trisaccharide structure as shown in Table $I$. 


\section{DISGUSSION}

The structures shown in Table I agreed with the results previously reported that isomaltotriose, ${ }^{11}$ panose, ${ }^{1 "}$ and $2^{G}$ - $\alpha$-glucosylcellobiose $^{2 !}$ were produced from isomaltose, maltose, and cellobiose, respectively, and that no addition of glucose occurred to $\alpha, \alpha$-trehalose." Beside these, now, the 1,6- $\alpha$-transglucosylation to the non-reducing unit could be shown in seven glucobioses, namely, $\alpha, \beta$-trehalose, $\beta, \beta$ trehalose, kojibiose, sophorose, nigerose, laminaribiose, and gentiobiose.

Isomaltose was the most effective acceptor of all glucobioses. This result agrees well with the fact that dextransucrase is a synthease of 1,6- $\alpha$-linked glucan. Maltose was the next efficient acceptor. Gentiobiose showed an efficiency of about half that of isomaltose. Among the three non-reducing glucobioses, only $\alpha, \alpha$-trehalose was inert, but it was found that $\alpha, \beta$ - and $\beta, \beta$-trehalose produced oligosaccharides in a moderate amount. $1,2-$ and 1 , 3-linked glucobioses were not so efficient acceptors.

A comparison between acceptors and trisaccharides produced indicated that a 1,2- $\alpha$ transglucosylation to reducing glucose unit occurred only in the case of cellobiose, and all the other glucobioses resulted in a 1,6- $\alpha$ transglucosylation to the non-reducing glucose unit.

Two non-reducing structures of trisaccharides from $\alpha, \beta$-trehalose were suggested, namely $1-\alpha$-isomaltosyl- $\beta$-glucoside and $1-\beta$-isomaltosyl- $\alpha$-glucoside. The former was produced by a 1,6- $\alpha$-transglucosylation to $\alpha$-glucosyl residue in $\alpha, \beta$-trehalose, and the latter to $\beta$-glucosyl residue. From the data in this experiment it cannot be distinguished whether the trisac- charide from $\alpha, \beta$-trehalose was single or an admixture as described above. The trisaccharide produced by the 1,6- $\alpha$-transglucosylation to $\beta, \beta$-trehalose only takes the structure as $1-\beta$-isomaltosyl- $\beta$-glucoside.

The produced trisaccharides of $1-\alpha$-isomaltosyl- $\beta$-glucoside or (and) $1-\beta$-isomaltosyl- $\alpha$-glucoside, 1 - $\beta$-isomaltosyl- $\beta$-glucoside, $2-\alpha$-isomaltosylglucose, 2 - $\beta$-isomaltosylglucose, $3-\beta$-isomaltosylglucose, and 6 - $\beta$-isomaltosylglucose, have never been reported, thus, these are new trisaccharides. More recently, 2- $\alpha$-isomaltosylglucose has also been isolated by us from the acetolyzate of a dextran ${ }^{18}$ and this showed exactly the same properties as described here. 3- $\alpha$-Isomaltosylglucose has been isolated from dextrans, ${ }^{19,201}$ hydrol, ${ }^{211}$ and the products formed by the action of T-enzyme to nigerose. ${ }^{22}$ The new 6 - $\beta$-isomaltosylglucose from gentiobiose is composed of two 1,6-linkages. One is $\alpha$ - and the other is $\beta$-configuration. A difference between this sugar and isomaltotriose was shown in paper chromatographic mobility (Fig. 2), $\beta$-glucosidase action, and specific optical rotation.

The structures of higher oligosaccharides, presumed to be 1,6- $\alpha$ homologous-series oligosaccharide containing the disaccharide of the acceptor at the reducing end, were suggested by the paper chromatographic mobility.

18) A forthcoming publication

19) I. J. Goldstein and W.J. Whelan, J. Chem. Soc., 1962, 170.

20) F. Yamauchi and K. Matsuda, Agr. Biol. Chem., 33, 103 (1969).

21) A. Sato and H. Ono, Chem. \& Ind., 1962, 1536.

22) M. Abdullah, I. J. Goldstein and W. J. Whelan, J. Chem. Soc., 1962, 176. 